\title{
Oral Manifestations of Chloroquine and Hydroxychloroquine: Differential Diagnoses
}

\author{
Samuel Rocha França*, Renan Ribeiro Benevides, Carlos Aragão Martins, \\ Ariana Vasconcelos Aragão, Filipe Nobre Chaves, \\ Denise Hélen Imaculada Pereira de Oliveira, Marcelo Bonifácio da Silva Sampieri
}

Federal University of Ceará, Campus Sobral, Sobral, Brazil

Email: ^samuelfranca@outlook.com

How to cite this paper: França, S.R., Benevides, R.R., Martins, C.A., Aragão, A.V., Chaves, F.N., de Oliveira, D.H.I.P. and da Silva Sampieri, M.B. (2020) Oral Manifestations of Chloroquine and Hydroxychloroquine: Differential Diagnoses. Open Journal of Stomatology, 10, 321-332.

https://doi.org/10.4236/ojst.2020.1011030

Received: September 8, 2020

Accepted: November 14, 2020

Published: November 17, 2020

Copyright $\odot 2020$ by author(s) and Scientific Research Publishing Inc. This work is licensed under the Creative Commons Attribution International License (CC BY 4.0).

http://creativecommons.org/licenses/by/4.0/

\begin{abstract}
Chloroquine phosphate and hydroxychloroquine sulfate are organic compounds, known as aminoquinolines for containing an amino group attached to a quinoline ring. They have been used since World War II as antimalarial agents. The article search that made up this review was carried out in the PubMed database, using the keywords "Chloroquine", "Hydroxychloroquine", and "Oral Manifestations", in the period including 2005 to 2020. The sample size was 7 female patients aged 40 to 66 years, with an age predominance of between 50 and 60 years old. The predominant lesion site was the hard palate with 6 cases. To reach the diagnosis of pigmented lesions in the oral cavity, meticulous anamnesis prior to physical examination is extremely important. In this pandemic and post-pandemic period, a more detailed investigation of the medications used by the patient in recent periods, such as chloroquine and hydroxychloroquine are essential to detect if the lesion was possibly caused by these drugs.
\end{abstract}

\section{Keywords}

Hydroxychloroquine, Chloroquine, Oral Manifestations, Hyperpigmentation, COVID-19

\section{Introduction}

Chloroquine phosphate and hydroxychloroquine sulfate are organic compounds, and known as aminoquinolines for containing an amino group attached to a quinoline ring. They have been used since World War II as antimalarial agents. Hydroxychloroquine sulfate is a derivative of chloroquine and was synthesized for the first time in 1946, by introducing a hydroxyl group [1] (Rainsford et al. 
2015).

Both drugs share the same mechanism of action. The compounds are fully and rapidly absorbed after oral administration and are excreted by the kidney and liver. Both are soluble in water, but for having a hydroxyl group, hydroxychloroquine is more soluble. The half-life is approximately $1-2$ months. The clinical safety profile of hydroxychloroquine is better than that of chloroquine and allows for higher daily doses with fewer concerns about drug interactions [2] (Gautret et al. 2020).

In addition, due to their immunosuppressive, anti-inflammatory, antithrombotic and metabolic actions, they have been widely and successfully used in the treatment of lupus erythematosus, rheumatoid arthritis, diabetes, cutaneous porphyria, HIV, fungal infections, immunological, dermatological, and infectious diseases, among others. Their antitumor properties stand out, which make these drugs an option in the treatment of various tumors associated with radio and chemotherapy. They also cross the blood-brain barrier, which makes them attractive as a therapeutic resource in neurological diseases [3] (Plantone et al. 2018).

Systemic administration of these drugs over a long period, can lead to multifocal hyperpigmentation on the skin and mucosa. Their cutaneous side effects include mild skin reactions, such as alopecia, itching, angioedema, rashes and pigmentation changes, and they are also known to exacerbate skin conditions such as psoriasis and porphyria. Cardiac and neuromuscular toxicities, together with retinopathy that can lead to blindness are also recognized as potential complications of their use [3] [4] (Plantone et al. 2018; Kalampalikis et al. 2012); as well as gastrointestinal manifestations, including nausea, vomiting and diarrhea. Furthermore, chloroquine or hydroxychloroquine overdose can cause acute intoxication and even death.

Of dental interest, oral hyperpigmentation induced by drug therapy can be attributed to stimulation of melanin production in melanocytes and/or tissue deposition of hemosiderin. Oral hyperpigmentation is reported mainly on the hard palate, but can be present in the gums, lips, and oral mucosa. It manifests as a well-defined pigmentation, brown, grayish blue or dark purple, in many sizes,being diffuse macular lesions with well-defined edges (Figure 1). The changes are usually reversible when medication is stopped or the dose is reduced, and treatment is not necessary. However, it is recommended that the patient be referred to their doctor, due to the risk of presenting other symptoms [4] [5] (Kalampalikis et al. 2012; de Filho et al. 2012).

Hyperpigmentation of the oral mucosa is common in melanoderm individuals (due to physiological deposition of melanin). Other conditions associated with hyperpigmentation of the oral mucosa include: melanocytic nevus, amalgam tattoo, and more rarely, melanoma; any of which can also present as an early sign of systemic disease. The dental surgeon must be aware of this context, in order to identify, treat, or otherwise avoid such effects whenever possible. 


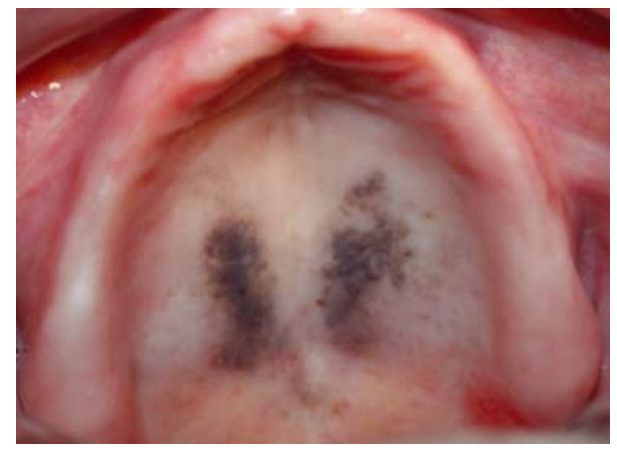

Figure 1. Hard palate hyperpigmentationusing chloroquine (ANDRADE et al. 2017).

Due to the greater toxicity that it presents in relation to hydroxychloroquine, most reports in the literature concerning oral manifestations due to the use of these drugs are related to chloroquine. Yet hydroxychloroquine also presents toxicity. Few cases of pigmentation attributed to chloroquine have been reported in the literature, and the incidence of hydroxychloroquine-induced pigmentation is estimated at between 7\% and 13\% [6] [7] (Sarma et al. 2020).

In December 2019, an outbreak of pneumonia began in Wuhan, China, spreading rapidly across the world. A new coronavirus (2019-nCoV) was identified, which is closely related to severe acute respiratory syndrome (SARS-CoV-2). The epidemic was declared a pandemic by the WHO on March 12, 2020. Faced with an urgent global pandemic, the scientific community looked to provide an effective treatment protocol for patients affected by COVID- 19 .

Among treatment options for COVID-19, chloroquine phosphate and hydroxychloroquine sulphate stand out. There is still much controversy about their efficacy and safety, which promotes great debate. Yet both chloroquine and hydroxychloroquine have been used in various treatment protocols and thousands of people have used these drugs. In view of this, the objective of this work is to evaluate side effects which can occur in the oral cavity due to the use of these drugs [8] [9] (Kleinegger et al. 2000).

\section{Methodology}

Thesearch for articles was carried out in the PubMed database, using the keywords "Chloroquine", "Hydroxychloroquine", and "Oral Manifestations", in the period including 2005 to 2020 . The article selection process involved analysis of titles and abstracts, restricted on the English language. Using the comination strategy in the "Chloroquine and Oral Manifestations" and "Hydroxychloroquine and Oral Manifestations", "COVID-19 and Oral manifestations", "COVID-19 and hyperpigmentation". Preclinical studies (such as in vitro studies, animal studies, and literature reviews) were excluded, yieldinga selection of 53 articles. Studies not presenting oral manifestations involving hydroxychloroquine or chloroquine were excluded, finally resulting in 7 studies being accepted (Figure 2). 


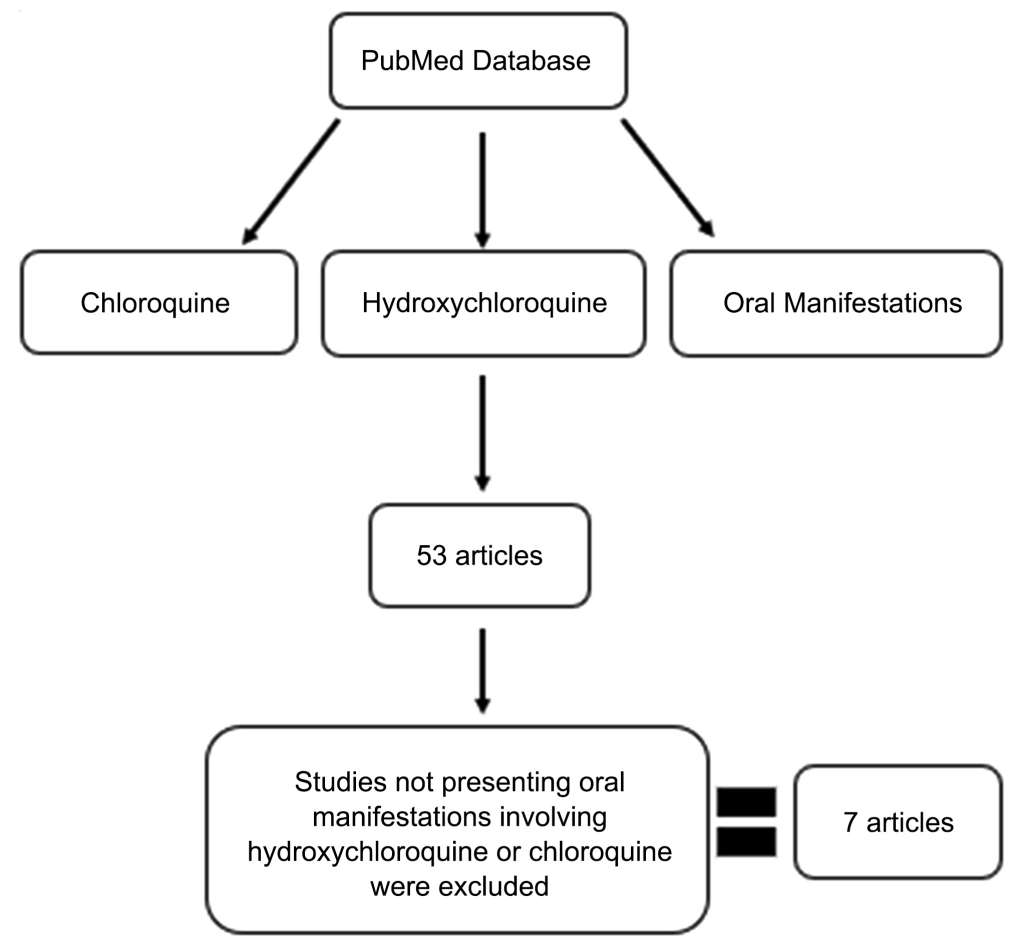

Figure 2. Literature search flow diagram.

\section{Results}

The results of the 7 studies are summarized in Table 1 . The sample size was 7 female patients aged 40 to 66 years, with an age predominance of between 50 and 60 years old [4] [10] [11]. The predominant lesion site was the hard palate with 6 cases [5] [10] [11] [12] [13] [14] and 1 patient with an injury manifested in the mandibular gingival region [4]. The dosages ranged from $150 \mathrm{mg}$ to 250 $\mathrm{mg}$ for chloroquine and from $200 \mathrm{mg}$ to $400 \mathrm{mg}$ for hydroxychloroquine. Chloroquine was the predominant drug reported [5] [11] [12] [13] [14]. The period of timeunder prescription for chloroquine ranged from 1 year to 15 years, and for hydroxychloroquine ranged from 8 months to 5 years. The predominant systemic disease, for which the use of the drug was justified, was rheumatoid arthritis [5] [10] [11] [12] [13] [14] with only one case related to Sjögren's syndrome [4]. Incisional biopsy for diagnostic confirmation of treatment was chosen in all cases. Interruption of the use of the medication [5] [10] [11] [12] followed. However, there were cases in which drug substitution [13] occurred and the injury was monitored [14].

\section{Discussion}

\subsection{Oral Manifestations}

When facing the injuries, we address in this study, the professional must adopt measures to optimize diagnosis and treatment. A detailed medical history with an accurate clinical examination is extremely important for elaboration of a diagnostic hypothesis. Due to the series of lesions presenting similar characteris- 
tics and yet different causes, the dentist can use an incisional biopsy for diagnostic confirmation [12] (ANDRADE et al. 2017), consistently with the conduct of all authors of the studies in Table 1 [4] [5] [10] [11] [12] [13] [14].

Another important aspect is managingthe use of these drugs, which needs to beperformed by the doctor (and patient) responsible for the treatment, analyzing the benefits and costs of using chloroquine and hydroxychloroquine in the medical treatment proposed. Thus, in some cases, it is possible to opt for a reduction in the usual dose or for atemporaryor definitive interruption altogether [12] (ANDRADE et al. 2017). The intensity of the effects and adverse reactions will dictate such maneuvers. It is worth mentioning that because the two drugs are in evidence in the current context of the pandemic of COVID-19, some protocols using these drugs are reporting using a higher dose than normally used for other diseases, leaving the question as to whether such doses can trigger earlier oral or even systemic manifestations in the patient. In the present study, we noted that the lowest dose was $150 \mathrm{mg}$ of chloroquine during a period of 1 year [12] and the highest dose was $400 \mathrm{mg}$ of hydroxychloroquine for 5 years [10]. As to time of use until an appearance of manifestations, we have that the period of 8 months [4] of hydroxychloroquine use and 15 years of chloroquine [5] use were the minimum and maximum times found in the studies in Table 1, revealing a great chronological variety in relation to the onset of hyperpigmentation in the oral cavity.

\subsection{Isolated Small Black, Brown, Brownish Lesions}

Pigmentations such as amalgam tattoo (Figure 3 ) and acquired melanocytic nevus (Figure 4), focal melanosis and oral melanoacanthoma are clinically smaller pigments that can be distinguished when taking a good medical history and performing exams. Small blackish pigments close to amalgam restorations are

Table 1. Studies presenting hydroxychloroquine and/or chloroquine oral manifestations.

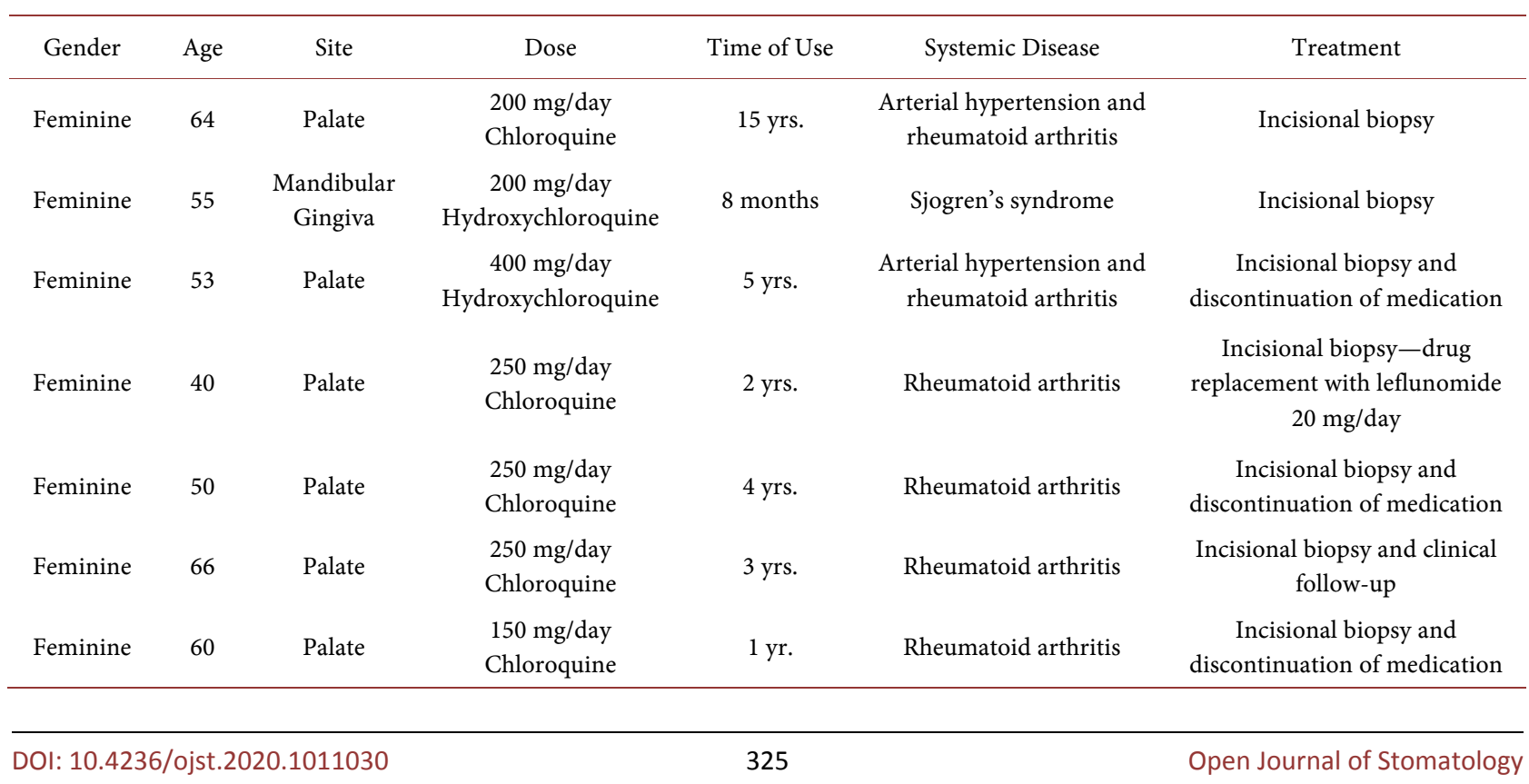




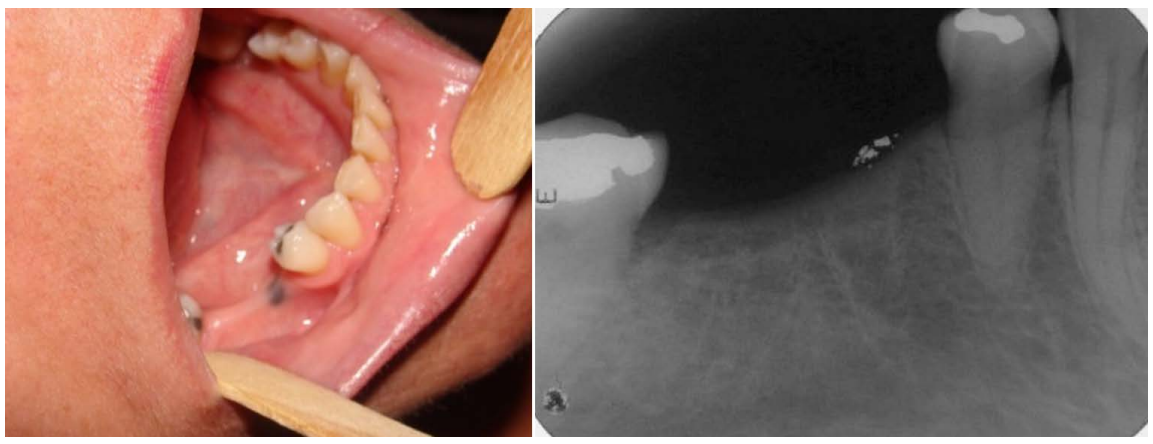

Figure 3. Amalgam tattoo, clinical and radiographic appearance (Stomatology clinic of UFC-Sobral).

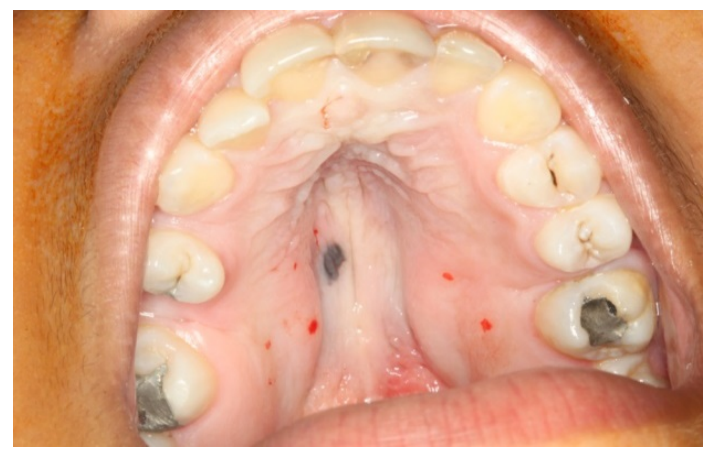

Figure 4. Acquired melanocytic nevus (Stomatology clinic of UFC-Sobral).

believed to be caused by the metallic material itself, a relatively common finding in the oral mucosa. Usually, it occurs when fragments of the restoration loosen and are incorporated into the gums when rubbing floss on newly restored teeth, or even during the performance of the restoration itself [15]. It manifests as a bluish or black isolated macula of small dimensions that when submitted to radiographic examination reveals a radiopacity [16] (Figure 3).

The clinical history of the pigmentation is important, since, during extraction, part of the amalgam restoration can fracture and fall into the open socket. This can lead to future amalgam pigmentation without the presence of the causative tooth. Observing these characteristics, the lesion is radiographed and if presenting a radiopaque area compatible with the pigmented region, the diagnosis of amalgam pigmentation is concluded [15] (Figure 3). In case of an absence of radiopacity; additional tests must follow.

If there is only small, isolated pigmentation, (discarding amalgam pigmentation after radiography), other lesions are considered, one of which is an acquired melanocytic nevus which consists of an accumulation of melanin in the skin that also affects the mouth (Figure 4). Benign melanocytic nevi begin to develop in childhood and have an evolution period of up to 35 years, entering a period of involution afterwards. The pigmentation affects both sexes and is more prevalent in white individuals [15]. For differential diagnosis, the patient's age, time of evolution, and skin tone are considered, these, given that the lesion, which is 
greater in childhood, presents slow and gradual growth which depends on racial differences [15], considering these and ruling out the hypothesis of possible malignant lesion, excisional biopsy is indicated for aesthetic purposes only.

The palate is a common site of pigmentation by chloroquine and hydroxychloroquine (Figure 1), and as seen in the Table1, the same is true for melanocytic nevus (Figure 4). Generally, nevus does not achieve large dimensions, unlike pigmentations by the medications in question. Itoccurs independently of any medication that the patient uses. A variant of the melanocytic nevus that draws attention is the blue nevus, which also consists of a benign proliferation of dermal melanocytes [15], yet these are in deeper layers of the skin, thus assuming a bluish tint that, when compared to pigmentation by anti-malarials, takes on a certain degree of distinction. Blue nevus is divided into two subtypes, common blue and cellular nevus, the distinction between them is in the size, where the first is smaller, varying in diameter between 1 to $5 \mathrm{~mm}$; while the second can reach sizes of larger than $5 \mathrm{~cm}$, which can resemble a chloroquine or hydroxychloroquine pigmentation (Figure 1), with prevalence in the palate, as is also the highest predilection for pigmentations caused by chloroquine and hydroxychloroquine (Table 1). Such lesions on the palate are a "warning light", and incisional biopsy is indicated. Depending on the histopathological result, treatment is determined. It is worth mentioning that there are controversies concerning potential risks of malignant transformation of blue nevus [17] [18].

Other dark and solitary pigments that can be found in the oral cavity are oral melanotic macula (focal melanosis) and oral melanoacanthoma (melanoacanthosis), which are acquired benign pigments. Both presentcharacteristics similar to pigmentation by the drugs in question, such as an oval or round macula, being asymptomatic, with brownish-yellow or dark brown color, and well-defined edges which stand out because they have a predilection for women, rapid proliferation. The involvement of melanoacanthosis that occurs almost exclusively in blacks with the focal melanosis presenting small dimensions [15]. Considering all of these clinical characteristics, and ruling out: a history of chronic smoking, patients with amalgam restoration, users of systemic medications with the potential for oral pigmentation, and those who do not have systemic disorders, it is the professional's responsibility to perform an excisional biopsy, or to periodically confirm the pigmentation, given its favorable prognosis. It is worth mentioning that due to the rapid and exaggerated growth of oral melanoacanthoma, incisional biopsy is indicated to rule out the possibility of melanoma [15].

\subsection{Multiple Small Black, Brown, Brownish Lesions}

\subsubsection{Peutz-Jeghers Syndrome}

This syndrome presents small dark brown-blue freckles, a few millimeters in diameter, occurring in the facial orifices, in the oral mucosa, and on the hands and feet. Pigmentation of the lips occurs more often (96\% of cases), followed by pigmentation of the oral mucosa ( $83 \%$ of cases) [19]. Peri-ocular, pigmentation 
is seen on the eyelids and along the eyelid margins, and, sometimes, on the eyelid conjunctiva [19], which differs from chloroquine and hydroxychloroquine pigmentation. Its characteristics are evident, since it can also be found in other regions of the body, and it is a hereditary manifestation differing from the pigmentation treated in this article, its intensity varies according to differences in gene expression.

The pigmentations can vary from intense and dispersed dark freckles to solitary pigmented patches [20] [21] [22] [23], developing in early childhood [15], unlike the studies involved in Table 1 where the average age was 55.5 years. Its diagnosis is made by exclusion, considering non-smoking patients, non-users of drugs with potential for oral pigmentation, and age. The presence of pigmentations in other areas of the body, as well as the evaluation of family history in relation to the disease must be verified, emphasizing the importance of physical examination of the entire mouth, lips, face, and limbs. Biopsy need not be considered unless there is doubt concerning the diagnosis.

\subsubsection{Racial Melanic Pigmentation}

Where there is excessive deposition of melanin in the melanocytes, leading to skin pigmentation, being common to black individuals, normal variation is considered. This is characterized by darkened and well-defined macules, mainly in the anterior buccal gingival region that usually present bilaterally, whether in small or large dimensions [15]. The location and predilection for black individuals are observed as differences between chloroquine and hydroxychloroquine pigmentation. Melanic pigmentation presents a certain degree of difference from other pigments, both because of its involvement, which is usually restricted to black individuals, and its history of evolution, which resembles that of a benign lesion. Once the diagnosis of racial pigmentation is complete, no treatment is necessary, except in patients seeking gingival aesthetics.

\subsubsection{Addison's Disease}

Another disease with manifestations of multiple, small and blackish pigments is Addison's disease; presenting as flat melanic pigments, diffuse, and mainly in the cheek mucosa. Differential diagnosis includes racial melanin pigmentation, tobacco-related pigmentation, drug-related pigmentation, type 1 neurofibromatosis, McCune-Albright syndrome and Peutz-Jeghers syndrome [21]. Addison's disease is differentiated from chloroquine and hydroxychloroquine pigmentation by its presentation of multiple small pigmentations. An exam for evaluation is the morning serum cortisol level, which if it results in values above 500 $\mathrm{nmol} / \mathrm{L}(18 \mu \mathrm{g} / \mathrm{dL})$, generally excludes Addison's disease, if below $165 \mathrm{nmol} / \mathrm{L}$ (6 $\mu \mathrm{g} / \mathrm{dL}$ ) it is suggestive of renal insufficiency. However, most patients will need a brief Sinac test to confirm or exclude Addison's disease. In this case, the incisional biopsy is of great help in the diagnosis, and in the case of a positive diagnosis for Addison's disease, the patient should be referred quickly for medical follow-up. Pigmented lesions are usually neglected, despite their severity. Pig- 
mented lesions can be isolated, multifocal, and of different sizes. Approaches for blackish, brown or brownish lesions differ according to the lesion's characteristics, since lesions with this aspect might turn out to be a malignant, such as melanoma. In a single small lesion $(<5 \mathrm{~mm})$ close to an amalgam restoration, it is good to request a radiographic examination to analyze the presence of radiopaque granules and, if they are present, the diagnosis of amalgam pigmentation is confirmed [22] (Meleti et al. 2008). If there is no radiopaque area, or if the lesion region is in the hard palate and/or the maxillary gingiva, incisional biopsy must be considered, since these are the most common sites for oral nevi and melanomas. In small multiple lesions with a likelihood of racial pigmentation, smoking, systemic conditions, or medication use, biopsy is not necessary (Figure 5) [22] [23] (Meleti et al. 2008; Hatch et al. 2005). In the case of large lesions, larger than $5 \mathrm{~mm}$ and located in the region of the palate and/or maxillary gingiva, it is essential to use the $A B C D$ technique to detect a possible melanoma (Figure 6) [24] (Elwood et al. 1996).

The technique consists of assessing the lesion and seeing if it has an " $\mathrm{A}$ " asymmetry in its presentation due to the uncontrolled growth of melanoma. A letter "B", has irregular edges, "C", presents coloration in hues, ranging from brown to black, and " $D$ ", presents a diameter greater than 5 - $6 \mathrm{~mm} \mathrm{[24]} \mathrm{[25]}$ [26] (Elwood et al. 1996; Thomas et al. 1998). If there is correlation in the evaluation, incisional biopsy is essential.

Flowchart: Approach to black, brown or brownish lesions

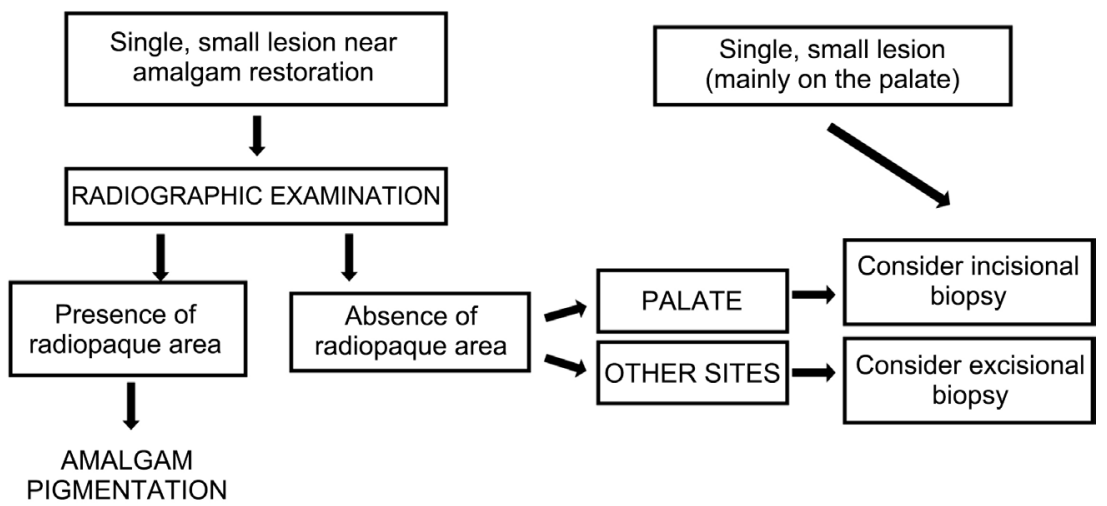

Figure 5. Algorithm for assessing pigmentation in the oral cavity.

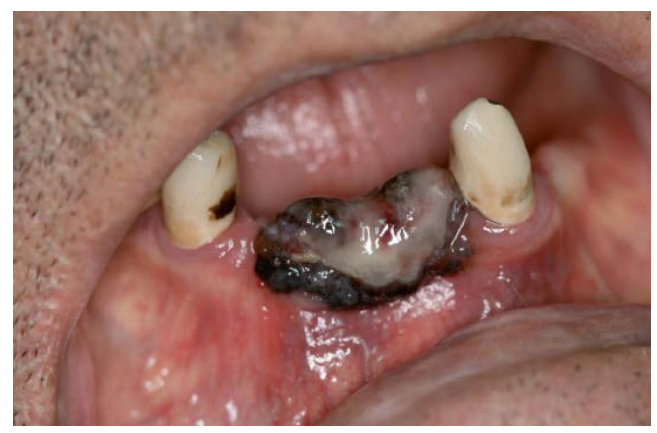

Figure 6. Melanoma in a rare site (MISIR et al. 2016). 


\section{Conclusion}

To arrive at a diagnosis for pigmented lesion in the oral cavity, meticulous anamnesis prior to physical examination is extremely important. In this pandemic and post-pandemic period, a more detailed investigation of the medications used by the patient in recent periods, such as chloroquine and hydroxychloroquine is essential to detect if the injury was possibly caused by these drugs. In case of doubt regarding the diagnosis, a biopsy is indicated.

\section{Conflicts of Interest}

The authors declare no conflicts of interest regarding the publication of this paper.

\section{References}

[1] Rainsford, K.D., Parke, A.L., Clifford-Rashotte, M. and Kean, W.F. (2015) Therapy and Pharmacological Properties of Hydroxychloroquine and Chloroquine in Treatment of Systemic Lupus Erythematosus, Rheumatoid Arthritis and Related Diseases. Inflammopharmacology, 23, 231-269. https://doi.org/10.1007/s10787-015-0239-y

[2] Gautret, P., Lagier, J.-C., Parola, P., Hoang, V.T., Meddeb, L., Mailhe, M., et al. (2020) Hydroxychloroquine and Azithromycin as a Treatment of COVID-19: Results of an Open-Label Non-Radomized Clinical Trial. International Journal of Antimicrobial Agents, 56, Article ID: 105949. https://doi.org/10.1016/j.ijantimicag.2020.105949

[3] PLantone, D. and Koudriavtseva, T. (2018) Current and Future Use of Chloroquine and Hydroxchloroquine in Infectious, Immune, Neoplastic, and Neurogical Diseases: A Mini-Review. Clinical Drug Investigation, 38, 653-671. https://doi.org/10.1007/s40261-018-0656-y

[4] Kalampalikis, A., Goetze, S. and Elsner, P. (2012) Isolierte Hyperpigmentierung der Mundschleimhaut durch Hydroxychloroquin. JDDG-Journal of the German Society of Dermatology, 10, 921-922. https://doi.org/10.1111/j.1610-0387.2012.08055 suppl.x

[5] de Filho, M.R.M., da Silva, C.A.D., da Dourado, M.R., de Pires, M.B.O., Pêgo, S.P.B. and de Freitas, E.M. (2012) Palate Hyperpigmentation Caused by Prolonged Use of the Anti-Malarial Chloroquine. Head \& Neck Pathology, 6, 48-50. https://doi.org/10.1007/s12105-011-0288-5

[6] Bahloul, E., Jallouli, M., Garbaa, S., Marzouk, S., Masmoudi, A., Turki, H., et al. (2017) Hydroxychloroquine-Induced Hyperpigmentation in Systemic Diseases: Prevalence, Clinical Features and Risk Factors: A Cross-Sectional Study of 41 Cases. Lupus, 26, 1304-1308. https://doi.org/10.1177/0961203317700486

[7] Sarma, P., Kaur, H., Kumar, H., Mahendru, D., Avti, P., Bhattacharyya, A., et al. (2020) Virological and Clinical Cure in COVID-19 Patients Treated with Hydroxychloroquine: A Systematic Review and Meta-Analysis. Journal of Medical Virology, 92, 776-785. https://doi.org/10.1002/jmv.25898

[8] Kleinegger, C.L., Hammond, H.L. and Finkelstein, M.W. (2000) Oral Mucosal Hyperpigmentation Secondary to Antimalarial Drug Therapy. Oral Surgery, Oral Medicine, Oral Pathology, Oral Radiology, and Endodontology, 90, 189-194.

https://doi.org/10.1067/moe.2000.106340 
[9] Moraes, P.C., Noce, C.W., Thomaz, L.A., Cintra, M.L. and Correa, M.E. (2011) Pigmented Lichenoid Drug Eruption Secondary to Chloroquine Therapy: An Unusual Presentation in Lower Lip. Minerva Stomatologica, 60, 327.

[10] Tosios, K.I., Kalogirou, E.M. and Sklavounou, A. (2018) Drug-Associated Hyperpigmentation of the Oral Mucosa: Report of Four Cases. Oral Surgery, Oral Medicine, Oral Pathology and Oral Radiology, 125, e54-e66.

https://doi.org/10.1016/j.000o.2017.10.006

[11] Manger, K., von Streitberg, U., Seitz, G., Kleyer, A. and Manger, B. (2018) Hard Palate Yperpigmentation-A Rare Side Effect of Antimalarials. Arthritis \& Rheumatology, 70, 152. https://doi.org/10.1002/art.40327

[12] Andrade, B., Padron-Alvarado, N., Munoz, C., Morais, T. and Martinez-Pedraza, R. (2017) Hyperpigmentation of Hard Palate Induced by Chloroquine Therapy. Journal of Clinical and Experimental Dentistry, 9, e1487-e1491.

https://doi.org/10.4317/jced.54387

[13] Ferrazzo, K.L., Payeras, M.R., Surkamp, P. and Danesi, C.C. (2017) Pathological Pigmentation of the Skin and Palate Caused by Continuous Use of Chloroquine: Case Report. Journal of Oral Diagnosis, 2. https://doi.org/10.5935/2525-5711.20170030

[14] Godinho, G.V., Paz, A.L.L.M., Araújo Gomes, E.P.A., Garcia, C.L. and Volpato, L.E.R. (2020) Extensive Hard Palate Hyperpigmentation Associated with Chloroquine Use. British Journal of Clinical Pharmacology, 86, 2325-2327.

[15] Neville, B.W., et al. (2016) Patologia oral e maxilofacial. 4th Edition, Elsevier, Rio de Janeiro, 912 p.

[16] Buchner, A. and Hansen, L.S. (1980) Amalgam Pigmentation (Amalgam Tattoo) of the Oral Mucosa. Oral Surgery, Oral Medicine, Oral Pathology, 49, 139-147. https://doi.org/10.1016/0030-4220(80)90306-0

[17] Pinto, A., Raghavendra, S., Lee, R., DeRossi, S. and Alawi, F. (2003) Epithelioid Blue Nevus of the Oral Mucosa: A Rare Histologic Variant. Oral Surgery, Oral Medicine, Oral Pathology, Oral Radiology, and Endodontology, 96, 429-436.

https://doi.org/10.1016/S1079-2104(03)00319-6

[18] Tran, T.A., Carlson, J.A., Basaca, P.C. and Mihm, M.C. (1998) Cellular Blue Nevus with Atypia (Atypical Cellular Blue Nevus): A Clinicopathologic Study of Nine Cases. Journal of Cutaneous Pathology, 25, 252-258. https://doi.org/10.1111/j.1600-0560.1998.tb01729.x

[19] Scott, R.J., Crooks, R., Meldrum, C.J., et al. (2002) Mutation Analysis of the STK11/ LKB1 Gene and Clinical Characteristics of an Australian Series of Peutz-Jeghers Syndrome Patients. Clinical Genetics, 62, 282-287. https://doi.org/10.1034/j.1399-0004.2002.620405.x

[20] Westerman, A.M. and Wilson, J.H.P. (1999) Peutz-Jeghers Syndrome: Risks of a Hereditary Condition. A Clinical Review. Scandinavian Journal of Gastroenterology, 34, 64-70. https://doi.org/10.1080/003655299750025561

[21] Chi, A.C., Neville, B.W., Krayer, J.W. and Gonsalves, W.C. (2010) Oral Manifestations of Systemic Disease, Medical University of South Carolina, Charleston, South Carolina. American Family Physician, 82, 1381-1388.

[22] Meleti, M., Vescovi, P., Mooi, W.J. and van der Waal, I. (2008) Pigmented Lesions of the Oral Mucosa and Perioral Tissues: A Flow-Chart for the Diagnosis and Some Recommendations for the Management. Oral Surgery, Oral Medicine, Oral Pathology, Oral Radiology, and Endodontology, 105, 606-616.

https://doi.org/10.1016/j.tripleo.2007.07.047 
[23] Hatch, C.L. (2005) Pigmented Lesions of the Oral Cavity. Dental Clinics of North America, 49, 185-201. https://doi.org/10.1016/j.cden.2004.07.013

[24] Elwood, J.M. (1996) Skin Self-Examination and Melanoma. Journal of the National Cancer Institute, 88, 3-5. https://doi.org/10.1093/jnci/88.1.3

[25] Thomas, L., Tranchand, P., Berard, F., Secchi, T., Colin, C. and Moulin, G. (1998) Semiological Value of ABCDE Criteria in the Diagnosis of Cutaneos Pigmented Tumors. Dermatology, 197, 11-17. https://doi.org/10.1159/000017969

[26] Mısır, A.F., Durmuşlar, M.C., Zerener, T. and Gün, B.D. (2016) Primary Malignant Melanoma. Saudi Medical Journal, 37, 446-449.

https://doi.org/10.15537/smj.2016.4.15017 